\title{
Vibrational response of functionally graded circular plate integrated with piezoelectric layers: An exact solution
}

\author{
A. A. Jandaghian ${ }^{\text {a }}$ A. A. Jafari ${ }^{\mathrm{b}}$ and O. Rahmani ${ }^{\mathrm{a}^{*}}$
}

${ }^{a}$ Smart Structures and New Advanced Materials Laboratory, Mechanical Engineering Department, University of Zanjan, Zanjan, Iran ${ }^{b}$ Faculty of Mechanical Engineering, K. N. Toosi University of Technology, Tehran. Iran

ART ICLE INFO

Article history:

Received September 20, 2013

Received in Revised form

October, 14, 2013

Accepted 30 January 2014

Available online

31 January 2014

Keywords:

Functionally graded material

Piezoelectric

Circular plate

Classical plate theory

Forced vibration

\begin{abstract}
A B S T R A C T
\end{abstract}
In this paper, harmonic forced vibration of circular functionally graded plate integrated with two uniformly distributed actuator faces made of piezoelectric material is studied. The material properties of the functionally graded substrate layers are assumed to be graded in the thickness direction according to the power-law distribution, also the distribution of electric potential field along the thickness direction of piezoelectric layers is modeled by a quadratic function. The governing equations are solved for simply supported boundary condition of the sandwich circular plate and the solutions are presented by elementary Bessel functions. The performance of the present model is compared with that of finite element analyses as well as other available literature by the presentation of comparative results obtained for several examples encompassing different power indexes and vibration modes. The results show that thickness of piezoelectric layer and changing the power index in FG material has a significant influence on the deflection and natural frequencies of system.

\section{Introduction}

The well-regulated vibrational properties of piezoelectric materials are broadly known as one of the most significant resources for the improvement in the smart structures. Intelligent self-monitoring and self-adaptive structures, incorporating piezoelectric patches, are now extensively used in the active and passive vibration control, in micro-electromechanical systems, in medical apparatus and in measuring devices. A metal substrate surface embedded or bonded by a piezoelectric sheet has received significant attention during last years for applied designs of actuators and sensors due to the electromechanically coupling characteristics.

* Corresponding author. Tel.: +989122414371; Fax: +982412283204

E-mail addresses: omid.rahmani@znu.ac.ir (Omid Rahmani) 
In recent times, functionally graded (FG) materials which exhibit smooth variation of material properties have been studied for developing intelligent functionally graded structures. By employing piezoelectric materials as sensors or actuators, intelligent FG structures have been made with proficiencies of self-monitoring and self-controlling. The design of systems including active piezoelectric materials requires comprehensive modeling of the mechanical, electrical and coupling properties of the piezoelectric elements, the host structure and their interactions. In the following recent progresses and findings in modeling of smart FG structures will be discussed.

Batra and coworkers (Batra \& Liang 1997; Vel \& Batra 2001) studied the vibration problem of a rectangular laminated plate with embedded piezoelectric actuators and sensors subjected to transient thermal loading. Batra and Geng (2002) considered a FG viscoelastic layer with a homogeneous PZT constraining layer and carried out the $3 \mathrm{D}$ transient analysis of the structure with the finite element method. He et al. (2001) proposed a FE formulation based on the classical laminated plate theory (CLPT) for the vibration and shape control of the FG material plates with integrated piezoelectric actuators and sensors and used a feedback control algorithm for the active control of the dynamic response of the plate through closed loop control. A FE formulation was proposed by Liew et al. (2002) for modeling and control of piezoelectric shell laminates under coupled temperature, displacement and electric potential fields. The base shell has been made of FG material which consists of combined metal- ceramic materials with different mixing ratios. Ootao and Tanigawa (2000) and Tanigawa (2001) investigated the FG simply supported plate integrated. A study on the nonlinear vibration and dynamic response of a FG material plate with surface-bonded piezoelectric layers in thermal environments was developed by Huang and Shen (2006). The nonlinear formulations were based on the higher-order shear deformation plate theory (HSDT) including thermo-piezoelectric effects. In this study, they accounted to heat conduction and temperaturedependent material properties, and it was assumed a variation through the plate thickness both for the temperature field and for the electrical field intensity.

A study on FG beams with surface integrated piezoelectric actuators and sensors based on a state space formulation was carried out by Bian et al. (2006). In their study, the bonding adhesive between the host beam and the piezoelectric layers was modeled by a spring layer in order to consider its effect. The bonding conditions were simulated through the consideration of different spring layer parameters. Free axisymmetric vibration problem of piezoelectric coupled thin circular and thin annular FGM plates has been carried out by Ebrahimi and Rastgoo (2008 a,b). Also Ebrahimi et al. (2009) suggested an analytical solution to analysis of smart moderately thick shear deformable and circular FG plate based on the Mindlin's plate theory. Wang and Quek (2001) studied free vibration of a circular plate surface bonded by two piezoelectric layers, based on the Kirchhoff theory. They have shown the mode shape of the electric potential obtained from free vibration analysis is generally to be non-uniform in the radial direction in contrast to what is commonly assumed. A study on the performance of vertically reinforced 1-3 piezoelectric composite distributed actuator in the active constrained layer damping system bonded to a FG plate was carried out by Ray and Batra (2007). They modelled the deformations of each layer by the first-order shear deformation theory. Jandaghian et al. (2013) suggested an analytical solution to investigate the transient motion of a circular plate surface bonded by two piezoelectric layers. By applying high-order sandwich panel theory (HSAPT) Rahmani et al. (2009) studied the free vibration of sandwich structure with a flexible functionally graded syntactic core. Yapeng (2003) presented an exact analysis of the free vibration of a functionally gradient piezoelectric plate. The solution of the derived governing differential equations was obtained through the power series expansion method. The natural frequencies and the modal distributions of free vibration of a functionally gradient piezoelectric plate were investigated.

In another study, Kargarnovin et al. (2007) investigated the active vibration control of FG material plates using piezoelectric sensor/actuator patches, using classical laminated plate (CLP) 
theory. In their work the effect of the feedback gain and the volume fraction on the plate frequency and displacement was studied. Es'haghi et al. (2011) presented an analytical solution for vibration study of piezoelectric coupled FG Mindlin plates those have open circuit piezoelectric patches and have been used as sensors. Fakhari et al. (2011) suggested a FE formulation based on HSDT plate theory to investigate the nonlinear natural frequencies, time and frequency responses of FG plate with surface-bonded piezoelectric layers under thermal, electrical and mechanical loads. Numerical results have been presented to study the effects of the volume fraction exponent, the applied voltage in piezoelectric layers, the thermal load and the vibration amplitude on nonlinear natural frequencies and time response of the plate with integrated piezoelectric layers was studied.

Hashemi et al. (2012) developed an analytical solution for the free vibration of piezoelectric coupled FG thick circular/annular plates on the basis of the Mindlin's FSDT theory and studied the effects of coupling between in-plane and transverse displacements on the frequency parameters. Loja et al. (2012) studied the static and free vibration behavior of functionally graded sandwich plate type structures, using B-spline finite strip element models based on different shear deformation theories. Geometrical nonlinear static and free vibration analyses of FG piezoelectric plates using FEM were studied by Behjat and Khoshravan (2012). On their work different sets of mechanical and electrical loadings were considered. The plate with FG piezoelectric material was considered to vary gradually through the thickness by a power law distribution. The electric potential was assumed to vary in a quadratic way through the thickness and was considered to be a nodal degree of freedom.

Liew et al (2003) presented a FE model for the static and dynamic piezothermoelastic analysis and control of FGM plates under temperature gradient environments using integrated piezoelectric sensor/actuator layers. They also applied a feedback control algorithm that couples the direct and inverse piezoelectric effects to provide active control of the integrated FGM plate in a closed loop system.

Along with the carried out literature review and to the best knowledge of authors there is no references for the forced vibration of FG circular plate integrated with piezoelectric material. In this study, the forced harmonic vibration of a FG thin circular plate integrated with piezoelectric layers is investigated for different boundary conditions. Both top and bottom layers of each piezoelectric layer are fully covered by electrodes which are shortly connected. The thickness of electrodes is considered to be extremely small compared to the plate thickness. Thus, in the following formulation, the mechanical effects of the electrodes are neglected. The properties of the substrate layers were graded in the thickness direction according to a volume fraction power law distribution. The distribution of electric potential field in the thickness direction of the piezoelectric layers is simulated by a quadratic function and the solutions are presented in terms of a single, elementary Bessel function. A consistent formulation based on the classical plate theory (CPT) that satisfies the Maxwell static electricity equation is presented for piezoelectric layers. The results are validated by those obtained from FEA and other available literature.

\section{Modeling of functionally graded properties}

The properties of the circular plate are considered to vary through the thickness of the plate with a power-law distribution of the volume fractions of the two materials in between the two surfaces. In fact, the top surface $(z=h)$ of the plate is ceramic-rich whereas the bottom surface $(z=-h)$ is metalrich. Poisson's ratio $v$ is assumed to be constant throughout the analysis. Young's modulus and mass density are assumed to vary continuously through the plate thickness as

$$
\begin{aligned}
& E(z)=\left(E_{c}-E_{m}\right) V_{f}(z)+E_{m}, \\
& \rho(z)=\left(\rho_{c}-\rho_{m}\right) V_{f}(z)+\rho_{m},
\end{aligned}
$$


where the subscripts $\mathrm{m}$ and $\mathrm{c}$ represent the metallic and ceramic constituents, respectively, $E$ and $\rho$ are modulus of elasticity and density, respectively and the volume fraction $V_{f}$ may be given by

$V_{f}=\left(\frac{z+h}{2 h}\right)^{g}, g \geq 0$

where $g$ is the power law index and takes only positive values. For $g=0$ and $g=1$, the plate is fully ceramic and metallic, respectively; whereas the composition of metal and ceramic is linear for $g=1$.

\section{Basic equations of FG sandwich circular plate with piezoelectric layers}

Consider a flat, thin FG sandwich circular plate, including one host layer in the middle and two identical piezoelectric faces bonded perfectly to the upper and lower surfaces of the host layer, with host plate thickness $2 h$ and piezoelectric layer thickness $h_{l}$, radius $r$, as illustrated in Fig. 1.

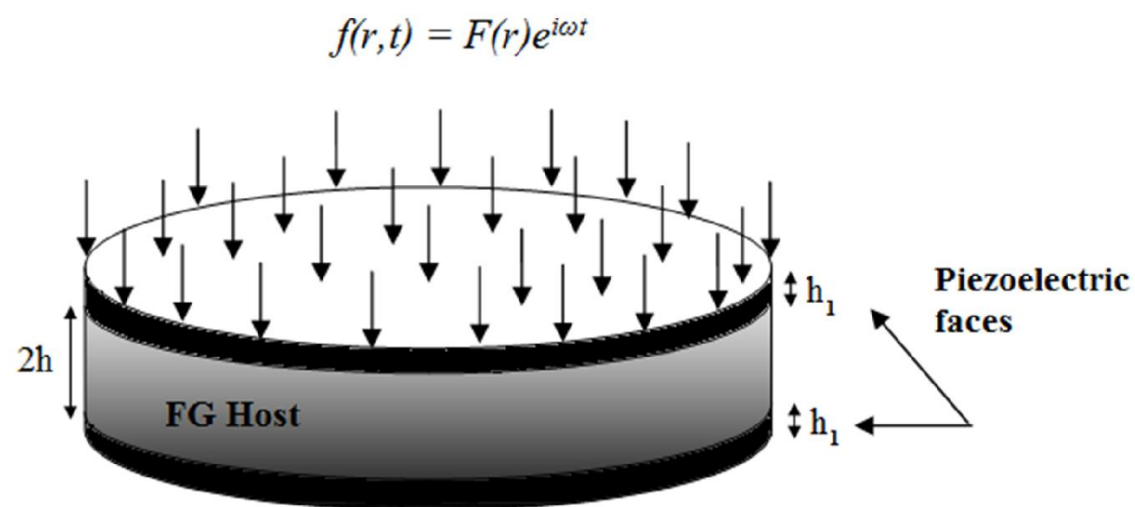

Fig. 1. Cross section of FGM circular plate with two piezoelectric layers mounted on its upper and lower surfaces

The constitutive equations for an piezoelectric layer in reference coordinate system $(z, r, \theta)$ are (Tiersten, 1969):

$$
\begin{aligned}
& \sigma_{r}^{p}=C_{11}^{E} \varepsilon_{r}+C_{12}^{E} \varepsilon_{\theta}+C_{13}^{E} \varepsilon_{z}-e_{31} E_{z}, \\
& \sigma_{\theta}^{p}=C_{12}^{E} \varepsilon_{r}+C_{11}^{E} \varepsilon_{\theta}+C_{13}^{E} \varepsilon_{z}-e_{31} E_{z}, \\
& \sigma_{z}^{p}=C_{13}^{E} \varepsilon_{r}+C_{13}^{E} \varepsilon_{\theta}+C_{33}^{E} \varepsilon_{z}-e_{33} E_{z},
\end{aligned}
$$

and $\varepsilon_{z}$ for plane stress is obtained from Eq. (6)

$\varepsilon_{z}=\frac{e_{33}}{C_{33}} E_{z}-\frac{C_{13}}{C_{33}}\left(\varepsilon_{r}+\varepsilon_{\theta}\right)$.

Substituting Eq. (7) into Eq. (4) and Eq. (5) gives

$$
\begin{aligned}
& \sigma_{r}^{p}=\left[C_{11}^{E}-\frac{\left(C_{13}^{E}\right)^{2}}{C_{33}^{E}}\right] \varepsilon_{r}+\left[C_{12}^{E}-\frac{\left(C_{13}^{E}\right)^{2}}{C_{33}^{E}}\right] \varepsilon_{\theta}-\left[e_{31}-\frac{C_{13}}{C_{33}} e_{33}\right] E_{z} \\
& \sigma_{\theta}^{p}=\left[C_{12}^{E}-\frac{\left(C_{13}^{E}\right)^{2}}{C_{33}^{E}}\right] \varepsilon_{r}+\left[C_{11}^{E}-\frac{\left(C_{13}^{E}\right)^{2}}{C_{33}^{E}}\right] \varepsilon_{\theta}-\left[e_{31}-\frac{C_{13}}{C_{33}} e_{33}\right] E_{z}
\end{aligned}
$$


$C_{11}^{E}$ and $C_{12}^{E}$ are the elastic modulus of the piezoelectric material in the radial and tangential directions, measured at constant electric field; and $e_{31}$ is the piezoelectric constant of the piezoelectric layer.

The stress components in the FG plate are expressed as (Timoshenko et al., 1959)

$$
\begin{aligned}
& \sigma_{r}^{f}=\frac{-z E(z)}{1-v^{2}}\left[w_{, r}+\frac{v w_{, r}}{r}\right], \\
& \sigma_{\theta}^{f}=\frac{-z E(z)}{1-v^{2}}\left[\frac{w_{, r}}{r}+v w_{, r r}\right], \\
& \tau_{r \theta}=0 .
\end{aligned}
$$

When the two major surfaces of the piezoelectric layer are held at zero voltage (i.e., closed circuit or boundary conditions), in this study we decided to adopt the following electric potential function which is appropriate for the proposed system (Wang et al., 2001):

$$
\varphi(r, z, t)=\left\{\begin{array}{l}
{\left[1-\left(\frac{2 z-2 h-h_{1}}{h_{1}}\right)^{2}\right] \phi(r, t), \quad h \leq z \leq h+h_{1}} \\
{\left[1-\left(\frac{-2 z-2 h-h_{1}}{h_{1}}\right)^{2}\right] \phi(r, t), \quad-h-h_{1} \leq z \leq-h}
\end{array}\right.
$$

where, $z$ is measured from the mid-plane of the plate in the global $z$-direction, $h_{1}$ is the thickness of the piezoelectric layer, and $\varphi(r, t)$ is the electric potential on the mid-surface of the piezoelectric layer. For boundary condition the electrodes on each piezoelectric layer are short-circuit and also, zero electrical potential at $r=a$ is obtained by connecting piezoelectric layers to ground. It is seen from Eq. (13) that the electric boundary condition of the short-circuit $(\phi=0)$ at the internal surfaces $z= \pm h$ and the external surfaces $z= \pm\left(h+h_{1}\right)$ of each piezoelectric layer is completely satisfied. The components of electric field intensity $E$ and electric flux density $D$ is written as (Wang et al., 2001)

$$
\begin{aligned}
& E_{r}=\left[1-4\left(\frac{z-h}{h_{1}}-\frac{1}{2}\right)^{2}\right] \frac{\partial \phi}{\partial r}, E_{z}=-\left(\frac{8(z-h)}{h_{1}^{2}}-\frac{4}{h_{1}}\right) \phi, E_{\theta}=0 \\
& D_{r}=-\bar{\Xi}_{11}\left[1-4\left(\frac{z-h}{h_{1}}-\frac{1}{2}\right)^{2}\right] \frac{\partial \phi}{\partial r}, D_{z}=\frac{8 \bar{\Xi}_{33}\left(z-h-h_{1} / 2\right)}{h_{1}^{2}} \phi+\bar{e}_{31}^{2} z \nabla^{2} w, D_{\theta}=0
\end{aligned}
$$

$E_{r}, E_{\theta}$ and $E_{z}$ are the electric field intensity in the $r, \theta$ and $z$ directions, respectively; $D_{r}, D_{\theta}$ and $D_{z}$ are the corresponding electric displacement; $\Xi_{11}$ and $\Xi_{33}$ are the dielectric constants of the piezoelectric layer; where $\bar{\Xi}_{11}$ and $\bar{\Xi}_{33}$ are reduced dielectric constants of the piezoelectric layer for the plane stress problem, which are given by $\bar{\Xi}_{11}=\Xi_{11}, \bar{\Xi}_{33}=\Xi_{33}+\left(e_{33}^{2} / C_{33}^{E}\right)$.

\section{Governing equations}

Afterward the resultant shear force is derived as:

$Q_{r}=M_{r, r}+\frac{M_{r}-M_{\theta}}{r}$ 
In addition, resultant components of sandwich circular plate are obtained as following:

$$
\begin{aligned}
& M_{r}=\int_{-h}^{h} z \sigma_{r}^{f} d z+2 \int_{h}^{h+h_{1}} z \sigma_{r}^{p} d z=-\left[\frac{4}{3} h_{1} \bar{e}_{31}+\left(v D_{1}+\frac{\bar{C}_{12}^{E}}{\bar{C}_{11}^{E}} D_{2}\right) \frac{w_{, r}}{r}+\phi\left(D_{1}+D_{2}\right) w_{, r r}\right], \\
& M_{\theta}=\int_{-h}^{h} z \sigma_{\theta}^{f} d z+2 \int_{h}^{h+h_{1}} z \sigma_{\theta}^{p} d z=-\left[\frac{4}{3} h_{1} \bar{e}_{31} \phi+\left(v D_{1}+\frac{\bar{C}_{12}^{E}}{\bar{C}_{11}^{E}} D_{2}\right) w_{, r r}+\left(D_{1}+D_{2}\right) \frac{w_{, r}}{r}\right],
\end{aligned}
$$

where the thickness of the main plate is $2 h$; the piezoelectric layer extends from $z=h$ to $z=h+h_{1}$. Substituting Eq. (17) into Eq. (18) and Eq. (16) and substituting the final results into the governing equation for the Kirchhoff plate

$Q_{r, r}+\frac{Q_{r}}{r}-\left(\int_{-h}^{h} \rho_{f}(z) \ddot{u}_{z} d z+2 \int_{h}^{h+h_{1}} \rho_{p} \ddot{u}_{z}\right)+f=0$.

where $f=f(r, t)$ is the external load will result in the equation for the piezoelectric sandwich $\mathrm{FG}$ circular plate:

$$
\left(D_{1}+D_{2}\right) \nabla^{4} w(r, t)+2\left(\bar{\rho} h+\rho_{p} h_{1}\right) \ddot{w}(r, t)+\frac{4}{3} h_{1} \bar{e}_{31} \nabla^{2} \phi(r, t)=f(r, t),
$$

where $\rho_{f}(z)$ and $\rho_{p}$ are material densities of the FG plate and piezoelectric layer, respectively. Also

$$
D_{1}=\frac{1}{1-v^{2}} \int_{-h}^{h} E(z) z^{2} d z, D_{2}=2 h_{1}\left(h^{2}+h h_{1}+\frac{h_{1}^{2}}{3}\right) C_{11}^{E} \quad \text { and } \quad \bar{\rho}=\frac{1}{2 h} \int_{-h}^{h} \rho_{f}(z) d z
$$

It should be noted that all of the electrical variables must satisfy Maxwell's equation which requires that the divergence of the electric flux density vanishes at any point within the media. This condition can be satisfied approximately by enforcing the integration of the electric flux divergence across the thickness of the piezoelectric layers to be zero for any $r$ and $z$ as (Wang et al., 2001)

$$
\int_{h}^{h+h_{1}}\left(D_{, r}+\frac{D_{r}}{r}+D_{, z} d z\right) d z=0
$$

Substituting Eqs. (15) into above equation yields

$$
\frac{h_{1}^{2} \bar{\Xi}_{11}}{12 \bar{\Xi}_{33}} \nabla^{2} \phi(r, t)+\frac{h_{1}^{2} \bar{e}_{31}^{2}}{8 \bar{\Xi}_{33}} \nabla^{2} w(r, t)-\phi(r, t)=0 .
$$

Finally, the problem leads to find the solution of two coupled PDEs (Eqs. 20 and 23) in association with the following electrical and mechanical boundary conditions and for fully grounded piezoelectric coupled, simply supported, plate

$$
\begin{aligned}
& z= \pm h: \varphi=0, \\
& z= \pm\left(h+h_{1}\right): \varphi=0 \\
& r=a: \varphi=0, w=M_{r}=0,
\end{aligned}
$$

\section{Forced vibration analysis}

The forcing function, $f(r, t)$, is assumed to be harmonic, with frequency $\omega$, as

$f(r, t)=F(r) e^{i \omega t}$ 
Thus, solutions of Eq. (20) and Eq. (23) are assumed to be of the form

$w(r, t)=W(r) e^{i \omega t}$,

$\varphi(r, t)=\varphi(r) e^{i \omega t}$,

where $W(r)$ is the amplitude in $z$ direction as a function of radial displacement only. $\varphi(r, t)$ is the electric potential on the mid-surface of the piezoelectric layer. Substituting Eq. (25) and Eq. (26) into Eq. (20) and Eq. (23) leads to

$$
\begin{aligned}
& \left(D_{1}+D_{2}\right) \nabla^{4} W(r)-2\left(\bar{\rho} h+\rho_{2} h_{1}\right) \ddot{W}(r) \omega^{2}+\frac{4}{3} h_{1} \bar{e}_{31} \nabla^{2} \phi(r)=F(r), \\
& \frac{h_{1}^{2} \bar{\Xi}_{11}}{12 \bar{\Xi}_{33}} \nabla^{2} \phi(r)+\frac{h_{1}^{2} \bar{e}_{31}^{2}}{8 \bar{\Xi}_{33}} W(r)-\phi(r)=0 .
\end{aligned}
$$

The final solution is combined of two solutions: (i) for the plate under the action of $F(r)$ and (ii) for the plate under the action of a radial bending moment applied at the edge. The complete solution is a suitable superposition of the two solutions, ensuring that the condition $M_{r}=0$ holds at $r=a$. The solution is therefore written in the form

$$
\begin{aligned}
& W(r)=\tilde{W}(r)+\bar{W}(r) \\
& \varphi(r)=\widetilde{\varphi}(r)+\bar{\varphi}(r)
\end{aligned}
$$

To find the first solution, we assume

$$
\begin{aligned}
& \widetilde{W}(r)=\sum_{j=1}^{\infty} A_{j} J_{0}\left(\alpha_{j} r\right), \\
& \widetilde{\varphi}(r)=\sum_{j=1}^{\infty} B_{j} J_{0}\left(\alpha_{j} r\right)
\end{aligned}
$$

In the following, $F(r)$ is expanded into a series in terms of Bessel functions, as

$F(r)=q_{1} J_{0}\left(\alpha_{1} r\right)+q_{2} J_{0}\left(\alpha_{2} r\right)+\ldots$

where

$q_{j}=\frac{2}{a^{2}} \frac{1}{J_{1}^{2}\left(\alpha_{j} a\right)} \int_{0}^{a} r q(r) J_{0}\left(\alpha_{j} r\right) d r, j=1,2, \ldots$

In Eq. (32), Eq. (33) and Eq. (34), $J_{0}(\alpha r)$ and $J_{1}(\alpha r)$ are Bessel functions of first kind of order zero and one, respectively, (Abramowitz \& Stegun 1964), and $\alpha_{j}$ are the positive roots of

$J_{0}(\alpha a)=0$

Substituting Eqs. (32), (33) into Eq. (23) gives

$$
B_{j}=\frac{-p_{2} \alpha_{j}^{2}}{1+\alpha_{j}^{2} p_{1}} A_{j}
$$

Substituting Eq. (32), Eq. (33) and Eq. (34) into Eq. (28) gives 


$$
A_{j}=\frac{q_{j}}{D\left(\alpha_{j}^{4}-\lambda^{4}+\frac{p_{0} p_{2} \alpha_{j}^{4}}{1+\alpha_{j}^{2} p_{1}}\right)}
$$

with $D=D_{1}+D_{2}$, and

$$
p_{0}=\frac{4 h_{1} \bar{e}_{31}}{3 D}, p_{1}=\frac{h_{1}^{2} \bar{\Xi}_{11}}{12 \bar{\Xi}_{33}}, p_{2}=\frac{h_{1}^{2} \bar{e}_{31}}{8 \bar{\Xi}_{33}}, \lambda^{4}=\frac{2\left(\bar{\rho} h+\rho_{2} h_{1}\right)}{D} \omega^{2}
$$

The solutions of $\widetilde{W}(r)$ and $\bar{W}(r)$ satisfy the boundary condition of $W(r=a)=0$, on the other hand they don't satisfy the boundary condition of $M_{r}=0$ at $r=a$ separately, but sum of them satisfy both conditions. Let the second solution, $\bar{W}(r)$ have the form:

$\bar{W}(r)=C\left(r^{2}-a^{2}\right)+W_{1}(r)$,

where constant $C$ and function $W_{l}(r)$ have to be calculated. Substituting Eq. (40) into Eq. (35) and Eq. (36) with $F(r)=0$, it is found that $W_{l}(r)$ and $\bar{\varphi}(r)$ must satisfy the following equations:

$$
\begin{aligned}
& \nabla^{4} W_{1}(r)+p_{0} \nabla^{2} \bar{\varphi}(r)-\lambda^{4}\left(C\left(r^{2}-a^{2}\right)+W_{1}(r)\right)=0, \\
& p_{0} \nabla^{2} \bar{\varphi}(r)-\bar{\varphi}(r)+p_{2} \nabla^{2} \bar{W}(r)=0 .
\end{aligned}
$$

The second solutions are expanded in the similar way as the first solutions.

$$
W_{1}(r)=\sum_{j=1}^{\infty} F_{j} J_{0}\left(\alpha_{j} r\right) \quad \bar{\varphi}(r)=\sum_{j=1}^{\infty} G_{j} J_{0}\left(\alpha_{j} r\right)
$$

Following similar steps than those used to derive $A_{j}$ and $B_{j}$, one gets

$$
\begin{aligned}
F_{j} & =\frac{\left(\frac{p_{0} p_{2} \bar{q}_{j} \alpha_{j}^{2}}{1+\alpha_{j}^{2} p_{1}}+c_{j}\right) C}{\left(\alpha_{j}^{4}-\lambda^{4}+\frac{p_{0} p_{2} \alpha_{j}^{4}}{1+\alpha_{j}^{2} p_{1}}\right)}, \\
G_{j} & =\frac{p_{2}\left(C \bar{q}_{j}-\alpha_{j}^{2} F_{j}\right)}{1+\alpha_{j}^{2} p_{1}},
\end{aligned}
$$

where

$$
\bar{q}_{j}=\frac{8}{a \alpha_{j} J_{1}\left(\alpha_{j} a\right)} \quad c_{j}=\frac{\lambda^{4} J_{2}\left(\alpha_{j} a\right)}{a \alpha_{j} J_{1}\left(\alpha_{j} a\right)}, j=1,2,
$$

The second solution $\widetilde{W}(r)$ and $\widetilde{\varphi}(r)$ satisfies Eqs. (28) and (29) with $F(r)=0$, and (24), except the boundary condition $M_{r}=0$ at $r=a$. substituting $\bar{W}(r)$ and $\tilde{W}(r)$ in this boundary condition

$$
r=a: \quad \bar{M}_{r}+\widetilde{M}_{r}=0
$$


Results in a linear, algebraic equation for $C$, whose value can easily be determined. Hence, the function $W(r)=\widetilde{W}(r)+\bar{W}(r)$ and $\varphi(r)=\widetilde{\varphi}(r)+\bar{\varphi}(r)$ are known and describe the exact, complete solution. Therefore the functions $W(r)$ and $\varphi(r)$ are known and describes the complete solution of the clamped boundary condition. Moreover, the final values of $w(r, t)$ and $\varphi(r, t)$ are:

$$
\begin{aligned}
& w(r, t)=\left(\sum_{j=1}^{\infty}\left(A_{j}+F_{j}\right) J_{0}\left(\alpha_{j} r\right)+C\left(r^{2}-a^{2}\right)\right) e^{i \omega t}, \\
& \varphi(r, t)=\left(\sum_{j=1}^{\infty}\left(B_{j}+G_{j}\right) J_{0}\left(\alpha_{j} r\right)\right) e^{i \omega t} .
\end{aligned}
$$

\section{Numerical results and discussion}

In the following vibrational response of simply supported circular sandwich plate subjected to a uniformly harmonic pressure all over the surface area is considered. The amplitude of external load is $1 \mathrm{kPa}$ and its frequency, $\omega$, is $100(\mathrm{rad} / \mathrm{s})$. The material parameters have been given in Table 1 . The thickness ratio of the piezoelectric layer and main plate $(h 1 / h)$ is $1 / 10$. The radius of circular plate is equal to $0.6 \mathrm{~m}$ and the thickness of FG host is $0.01 \mathrm{~m}$.

Table 1. Material properties and geometric size of the piezoelectric coupled FGM plate (Wang. et al. 2001)

\begin{tabular}{ccc}
\hline & Host FG plate & \\
\hline$E_{c}=205(\mathrm{GPa})$ & $\rho_{c}=7800\left(\mathrm{~kg} / \mathrm{m}^{3}\right)$ \\
$E_{m}=200(\mathrm{GPa})$ & $\rho_{m}=8900\left(\mathrm{~kg} / \mathrm{m}^{3}\right)$ \\
\hline & PZT & \\
\hline$C_{11}^{E}=132 \times 10^{9}$ & $C_{12}^{E}=71 \times 10^{9}$ \\
$C_{33}^{E}=115 \times 10^{9}$ & $C_{13}^{E}=73 \times 10^{9}$ \\
$e_{31}=-4.1\left({\left.\mathrm{C} / \mathrm{m}^{2}\right)}^{-9}(\mathrm{~F} / \mathrm{m})\right.$ & $e_{33}=14.1\left(\mathrm{C} / \mathrm{m}^{2}\right)$ \\
$\Xi_{11}=7.124 \times 10^{-9}(\mathrm{~F} / \mathrm{m})$ \\
\end{tabular}

Fig.2. shows the radial bending moment of plate of plate and Fig. 3 shows the radial distribution of the deflection at time $t=0.2(\mathrm{~s})$ for $g=0$ (isotropic plate). The curves in Figs. 2 and 3 show that boundary conditions at $r=a: w=M_{r}=0$ are satisfied. Since there were no published results for forced vibration of piezo-FG circular sandwich plate, the obtained results have been verified with those obtained from Abaqus results. Fig. 4 shows the vibration of compound piezo-plate centre respect to time. As seen from Fig. 2 and Fig. 4 there is a good agreement between curve obtained from our method and curve obtained from FEM.

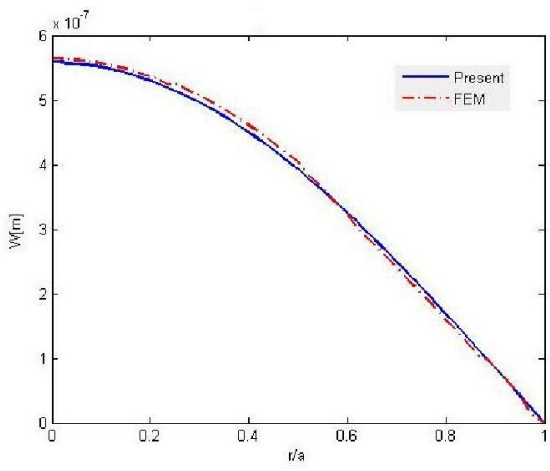

Fig. 2. Radial distribution of deflection of PiezoFG plate at $t=0.2(\mathrm{~s})$ for $g=0$

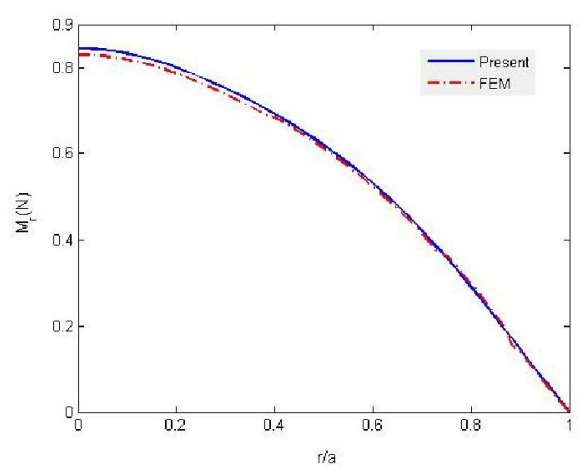

Fig. 3. Radial bending moment of Piezo-FG plate at $\mathrm{t}=0.2(\mathrm{~s})$ for $g=0$ 
Table 2 shows the centre deflection of piezo-FG plate at time $t=0.7 \mathrm{~s}$ for different $g$ under the same value of mechanical load. As seen from Table 2 the deflection of piezo-plate decreases by increasing of $g$ and obtained results from our method correspond with the results of FEM solution. In order to see better the effect of $g$ variations on the deflection of plate, Fig. 5 also illustrate these variations.

Table 2. Effect of power index (g) on the deflection of plate

\begin{tabular}{c|ccc}
\multirow{2}{*}{$\begin{array}{c}\text { Power } \\
\text { Index }(\mathrm{g})\end{array}$} & Present & FEM & Diff $(\%)$ \\
\cline { 2 - 4 } & $4.319 \mathrm{E}-07$ & $4.345 \mathrm{E}-07$ & -0.61 \\
\hline 0 & $4.280 \mathrm{E}-07$ & $4.300 \mathrm{E}-07$ & -0.47 \\
\hline 1 & $4.271 \mathrm{E}-07$ & $4.280 \mathrm{E}-07$ & -0.21 \\
\hline 5 & $4.267 \mathrm{E}-07$ & $4.275 \mathrm{E}-07$ & -0.19 \\
\hline 7 & $4.263 \mathrm{E}-07$ & $4.270 \mathrm{E}-07$ & -0.16 \\
\hline 9 & $4.260 \mathrm{E}-07$ & $4.266 \mathrm{E}-07$ & -0.14 \\
\hline
\end{tabular}

Note that as the forcing frequency, $\omega$, approaches the $j$ th frequency of vibration of the plate, $\left[\frac{D}{2\left(\bar{\rho} h+\rho_{2} h_{1}\right)}\left(1+\frac{p_{0} p_{2}}{1+\alpha_{j}^{2} p_{1}}\right)\right]^{1 / 2} \alpha_{j}^{2}$, in Equations (30) and (35) the deflection of the plate $W(r) \rightarrow \infty$ , thereby causing resonance. Table 3 shows the first three axisymmetric natural frequencies of piezoFG plate for different values of $g$. The obtained results in Table 3 indicate that by increasing the value of $g$, the frequency of system decreases. Moreover, this decreasing trend of frequency for smaller values of $g$ is more pronounced, for example by increasing the value of $g$ from 1 to 3 ( $200 \%$ ), the frequency of the first mode for the compound plate decreases by $1.32 \%$ but by increasing $g$ from 3 to $9(\sim 200 \%)$ of the same plate and for the same mode, the frequency decreases by $0.71 \%$. In order to see better the effect of $g$ variations on the natural frequency of the different plates, Figs. 6 to 8 also illustrate these variations for the first three mode shape.

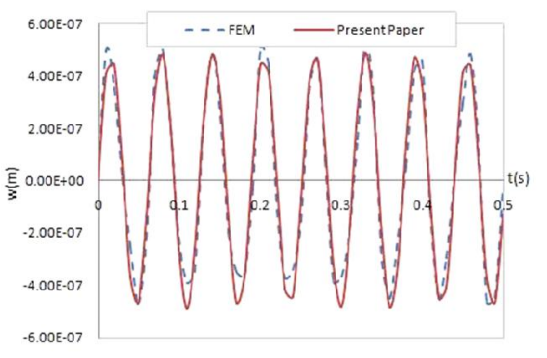

Fig. 4. Dynamic deflection of plate at $r$ $=0$ for $g=0$

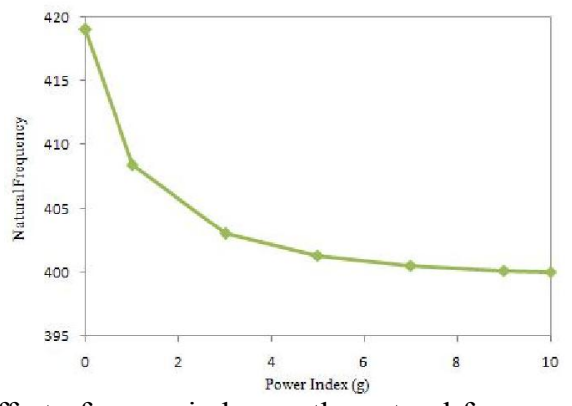

Fig. 7. Effect of power index on the natural frequency (second mode)

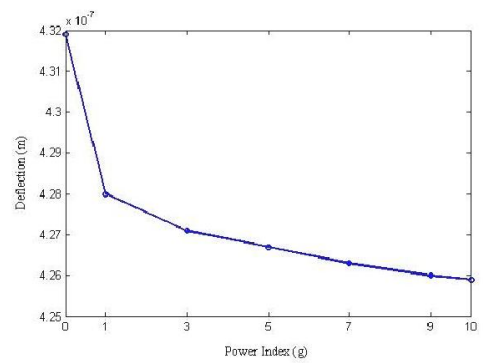

Fig 5. Effect of power index $(g)$ on deflection

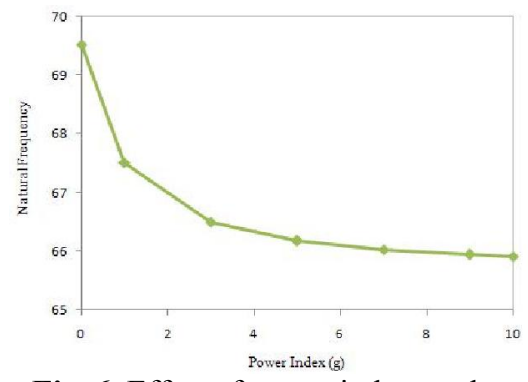

Fig. 6. Effect of power index on the natural frequency (First mode)

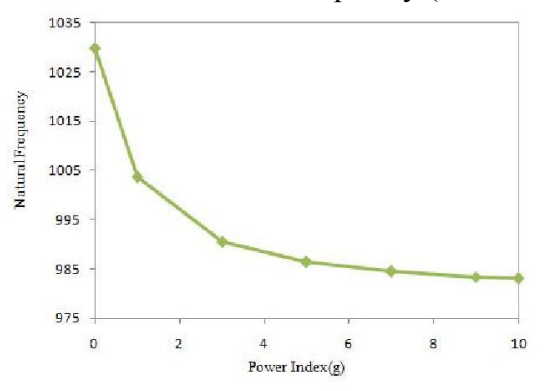

Fig. 8. Effect of power index on the natural frequency (Third mode) 
Table 3. The First three modes resonance frequencies for Piezo-FG plate for various values of power index

\begin{tabular}{|c|c|c|c|c|c|}
\hline Power Index & Mode no. & Present $(\mathrm{Hz})$ & Ebrahimi and Rastgo (2008a) & $\operatorname{Diff}(\%)$ & Wang et al. (2001) \\
\hline \multirow[t]{3}{*}{ - } & 1 & 69.52 & 69.37 & 0.22 & 69.33 \\
\hline & 2 & 418.99 & 417.86 & 0.27 & 418.03 \\
\hline & 3 & 1029.74 & 1039 & -0.9 & 1038.2 \\
\hline \multirow{3}{*}{1} & 1 & 67.51 & 67.88 & -0.55 & - \\
\hline & 2 & 408.38 & 406.83 & 0.38 & - \\
\hline & 3 & 1003.65 & 1001.51 & 0.21 & - \\
\hline \multirow{3}{*}{3} & 1 & 66.49 & 67.07 & -0.87 & - \\
\hline & 2 & 403.01 & 402.23 & 0.19 & - \\
\hline & 3 & 990.45 & 983.5 & 0.19 & - \\
\hline \multirow{3}{*}{5} & 1 & 66.17 & 66.81 & -0.97 & - \\
\hline & 2 & 401.33 & 400.71 & 0.15 & - \\
\hline & 3 & 986.33 & 982.2 & 0.42 & - \\
\hline \multirow{3}{*}{7} & 1 & 66.02 & 66.72 & -1.06 & - \\
\hline & 2 & 400.55 & 397.32 & 0.81 & - \\
\hline & 3 & 984.42 & 980.4 & 0.41 & - \\
\hline \multirow{3}{*}{9} & 1 & 65.94 & 66.61 & -1.02 & - \\
\hline & 2 & 400.12 & 396.81 & 0.83 & - \\
\hline & 3 & 983.36 & 979.02 & 0.44 & - \\
\hline \multirow{3}{*}{10} & 1 & 65.91 & 66.59 & -1.03 & - \\
\hline & 2 & 399.67 & 396.5 & 0.79 & - \\
\hline & 3 & 982.98 & 978.1 & 0.5 & - \\
\hline
\end{tabular}

As seen from Fig. 7, the behavior of the system follows the same trend in all different cases, i.e. the natural frequency of the system decrease by increasing of $g$ and stabilizes for $g$ values greater than 7. In fact for $g>>1$, the FGM plate becomes a ceramic plate and the compound plate transforms into a laminated plate with ceramic core as s host plate. Table 4 shows the effect of piezoelectric layer on the natural frequency system. As seen from Table 4 by increasing piezoelectric layer, the natural frequency increases and the effect of adding piezoelectric layers is more obvious when there is a thicker piezoelectric layer on the main plate.

Table 4. Effect of piezoelectric layer on resonance frequencies

\begin{tabular}{cccc}
\hline Thickness ratio & First mode & Second mode & Third mode \\
\hline $1 / 12$ & 68.65 & 417.62 & 1026.62 \\
$1 / 10$ & 69.52 & 418.99 & 1029.74 \\
$1 / 8$ & 71.52 & 420.96 & 1034.82 \\
$1 / 5$ & 73.68 & 428.44 & 1052.90 \\
\hline
\end{tabular}

\section{Conclusion}

A model for the analysis the forced vibration of a piezoelectric coupled FG circular plate structure is proposed. The equation of motion achieved based on the CPT for harmonic vibration. The solutions are given in terms of elementary Bessel functions and validation is done using the results from present method and those from FEA. It is shown that the thickness of piezo-layer has significant effect on the deflection amplitude and natural frequency of piezo-FG plate. Also power index $(g)$ has significant effect on the deflection amplitude and natural frequency of piezo-FG sandwich plate.

\section{References}

Abramowitz, M., \& Stegun, I. A. (Eds.). (1964). Handbook of Mathematical Functions: With Formulars, Graphs, and Mathematical Tables (Vol. 55). DoverPublications.com.

Batra, R. C., \& Geng, T. S. (2002). Comparison of active constrained layer damping by using extension and shear mode piezoceramic actuators. Journal of Intelligent Material Systems and Structures, 13(6), 349-367.

Batra, R. C., \& Liang, X. Q. (1997). The vibration of a rectangular laminated elastic plate with embedded piezoelectric sensors and actuators. Computers \& structures, 63(2), 203-216.

Behjat, B., \& Khoshravan, M. R. (2012). Geometrically nonlinear static and free vibration analysis of functionally graded piezoelectric plates. Composite Structures, 94(3), 874-882. 
Bian, Z. G., Lim, C. W., \& Chen, W. Q. (2006). On functionally graded beams with integrated surface piezoelectric layers. Composite structures, 72(3), 339-351.

Ebrahimi, F., \& Rastgo, A. (2008a). An analytical study on the free vibration of smart circular thin FGM plate based on classical plate theory. Thin-Walled Structures, 46(12), 1402-1408.

Ebrahimi, F., \& Rastgoo, A. (2008b). Free vibration analysis of smart annular FGM plates integrated with piezoelectric layers. Smart Materials and Structures, 17(1), 015044.

Ebrahimi, F., Rastgoo, A., \& Atai, A. A. (2009). A theoretical analysis of smart moderately thick shear deformable annular functionally graded plate. European Journal of Mechanics-A/Solids, 28(5), 962-973.

Es' haghi, M., Hashemi, S. H., \& Fadaee, M. (2011). Vibration analysis of piezoelectric FGM sensors using an accurate method. International Journal of Mechanical Sciences, 53(8), 585-594.

Fakhari, V., Ohadi, A., \& Yousefian, P. (2011). Nonlinear free and forced vibration behavior of functionally graded plate with piezoelectric layers in thermal environment. Composite Structures, 93(9), 2310-2321.

Hashemi, S. H., Khorshidi, K., Es'haghi, M., Fadaee, M., \& Karimi, M. (2012). On the effects of coupling between in-plane and out-of-plane vibrating modes of smart functionally graded circular/annular plates. Applied Mathematical Modelling, 36(3), 1132-1147.

He, X. Q., Ng, T. Y., Sivashanker, S., \& Liew, K. M. (2001). Active control of FGM plates with integrated piezoelectric sensors and actuators. International Journal of Solids and Structures, 38(9), 1641-1655.

Huang, X. L., \& Shen, H. S. (2006). Vibration and dynamic response of functionally graded plates with piezoelectric actuators in thermal environments. Journal of Sound and Vibration, 289(1), 25-53.

Jandaghian, A. A., Jafari, A. A., \& Rahmani, O. (2013). Transient Bending of a Circular Plate Integrated with Piezoelectric layers. Applied Mathematical Modelling,37(12-13), 7154-7163.

Kargarnovin, M. H., Najafizadeh, M. M., \& Viliani, N. S. (2007). Vibration control of a functionally graded material plate patched with piezoelectric actuators and sensors under a constant electric charge. Smart materials and structures, 16(4), 1252.

Liew, K. M., He, X. Q., Ng, T. Y., \& Kitipornchai, S. (2002). Active control of FGM shells subjected to a temperature gradient via piezoelectric sensor/actuator patches. International journal for numerical methods in engineering, 55(6), 653-668.

Liew, K. M., He, X. Q., Ng, T. Y., \& Kitipornchai, S. (2003). Finite element piezothermoelasticity analysis and the active control of FGM plates with integrated piezoelectric sensors and actuators. Computational Mechanics, 31(3-4), 350-358.

Loja, M. A. R., Soares, M., \& Barbosa, J. I. (2013). Analysis of functionally graded sandwich plate structures with piezoelectric skins, using B-spline finite strip method. Composite Structures, 96, 606-615.

Ootao, Y., \& Tanigawa, Y. (2000). Three-dimensional transient piezothermoelasticity in functionally graded rectangular plate bonded to a piezoelectric plate. International Journal of Solids and Structures, 37(32), 4377-4401.

Rahmani, O., Khalili, S. M. R., Malekzadeh, K., \& Hadavinia, H. (2009). Free vibration analysis of sandwich structures with a flexible functionally graded syntactic core. Composite Structures, 91(2), 229-235.

Ray, M. C., \& Batra, R. C. (2007). Vertically reinforced 1-3 piezoelectric composites for active damping of functionally graded plates. AIAA journal, 45(7), 1779-1784.

Tanigawa, Y. (2001). Control of the transient thermoelastic displacement of a functionally graded rectangular plate bonded to a piezoelectric plate due to nonuniform heating. Acta mechanica, 148(1-4), 17-33.

Tiersten, H. F. (1969). Linear piezoelectric plate vibrations: Elements of the linear theory of piezoelectricity and the vibrations of piezoelectric plates. Plenum Press.

Timoshenko, S., \& Woinowsky-Krieger, S. (1959). Theory of plates and shells (Vol. 2, pp. 240-246). New York: McGraw-hill.

Vel, S. S., \& Batra, R. C. (2001). Exact solution for rectangular sandwich plates with embedded piezoelectric shear actuators. AIAA journal, 39(7), 1363-1373.

Wang, Q., Quek, S. T., Sun, C. T., \& Liu, X. (2001). Analysis of piezoelectric coupled circular plate. Smart Materials and Structures, 10(2), 229.

Yapeng, W.X.S. (2003) Three-dimensional solution for the free vibration of functionally gradient piezoelectric plates, Acta Mechanica Solida Sinica 1:010 